\title{
Shutdown Dose Calculations for the IFMIF-DONES Lithium Loop Cell Using Variance Reduction Techniques
}

\author{
Yuefeng Qiu*, Ulrich Fischer
}

\section{Introduction}

- IFMIF-DONES is a DEMO Oriented NEutron Source providing the irradiation data needed for the construction of DEMO. The Lithium Loop Cell (LLC) is a room below the test cell (TC) housing the equipment for the lithium system.

The shutdown dose from the activated components in the TC is a concern for the maintenance and radiation safety in the LLC.
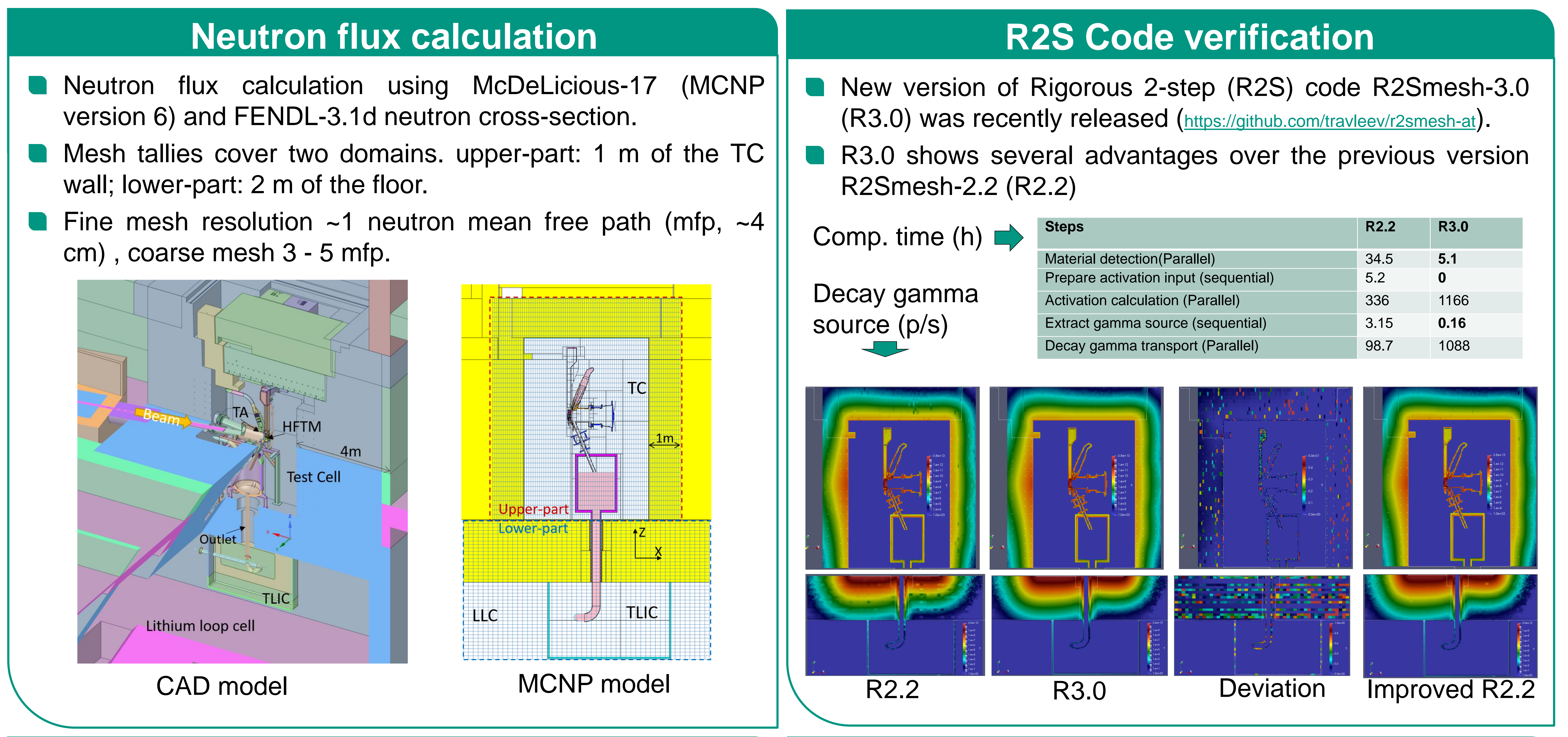

\section{Variance reduction}

- Intensity based source sampling method in the "Common R2S" package was used, In order to obtain constant source particle weight.

- ADVANTG code was used to produce a WWM using a 1 $\mathrm{MeV}$ uniform gamma source in the first $0.5 \mathrm{~m}$ layer of the inner TC shielding.

- Statistics of dose results in the TLIC is improved, the dose rate outside the TLIC is $<0.5 \mu \mathrm{Sv} / \mathrm{h}$.

Uniform sampling, source weight $=$ gamma intensity
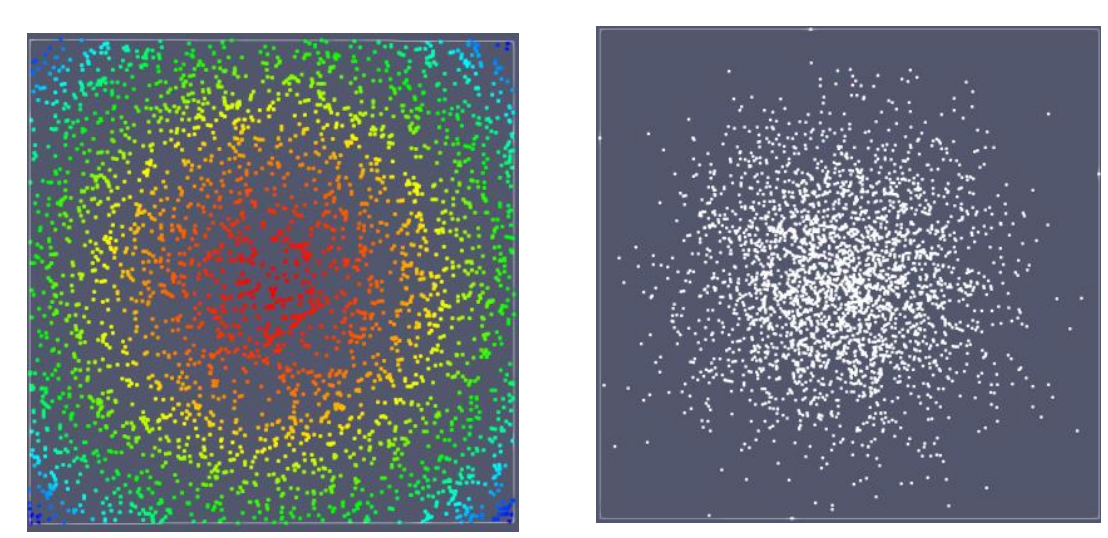

Source intensity based sampling, source weight $=1$ associated with gamma intensity.

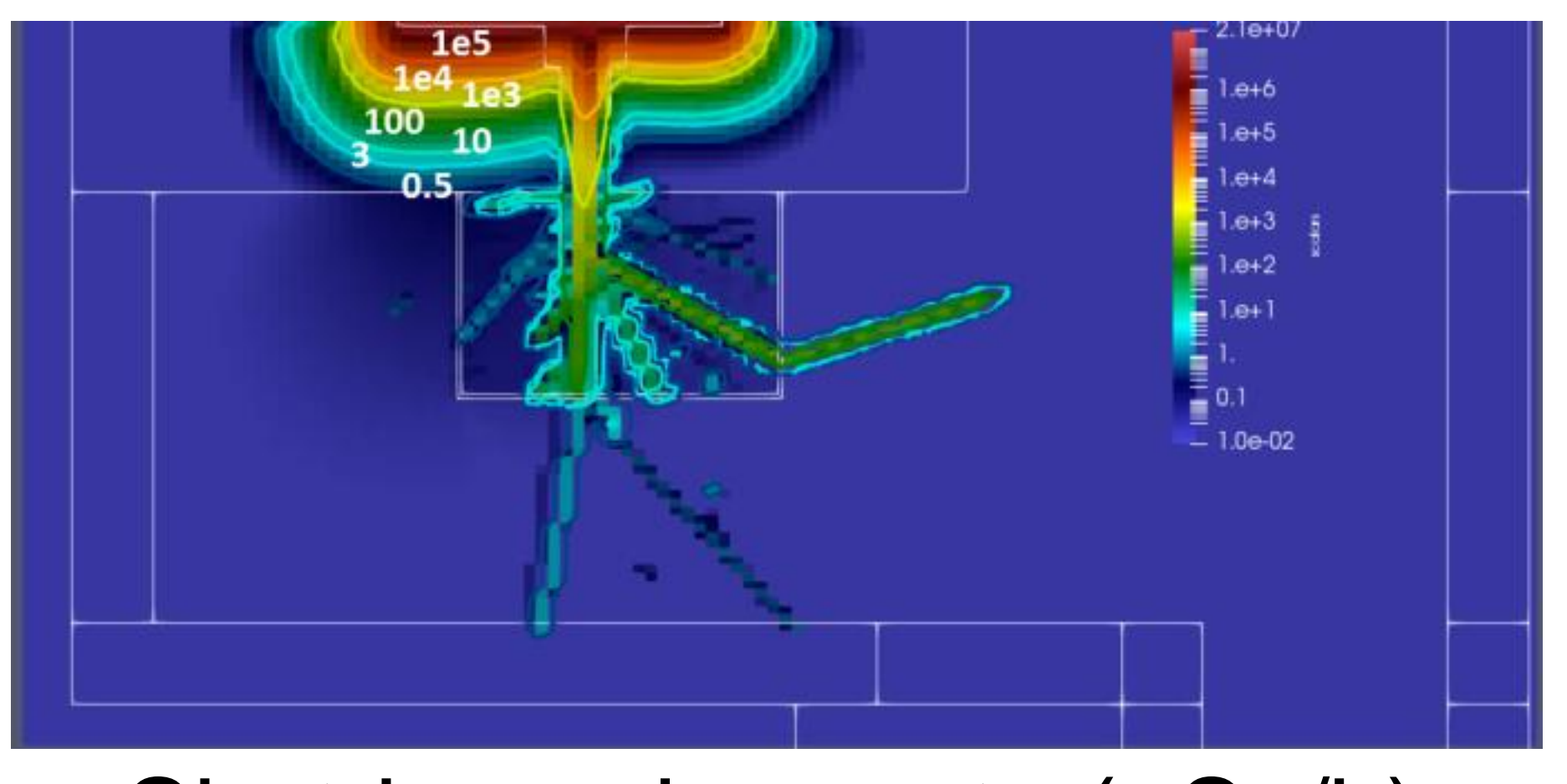

Shutdown dose rate $(\mu \mathrm{Sv} / \mathrm{h})$

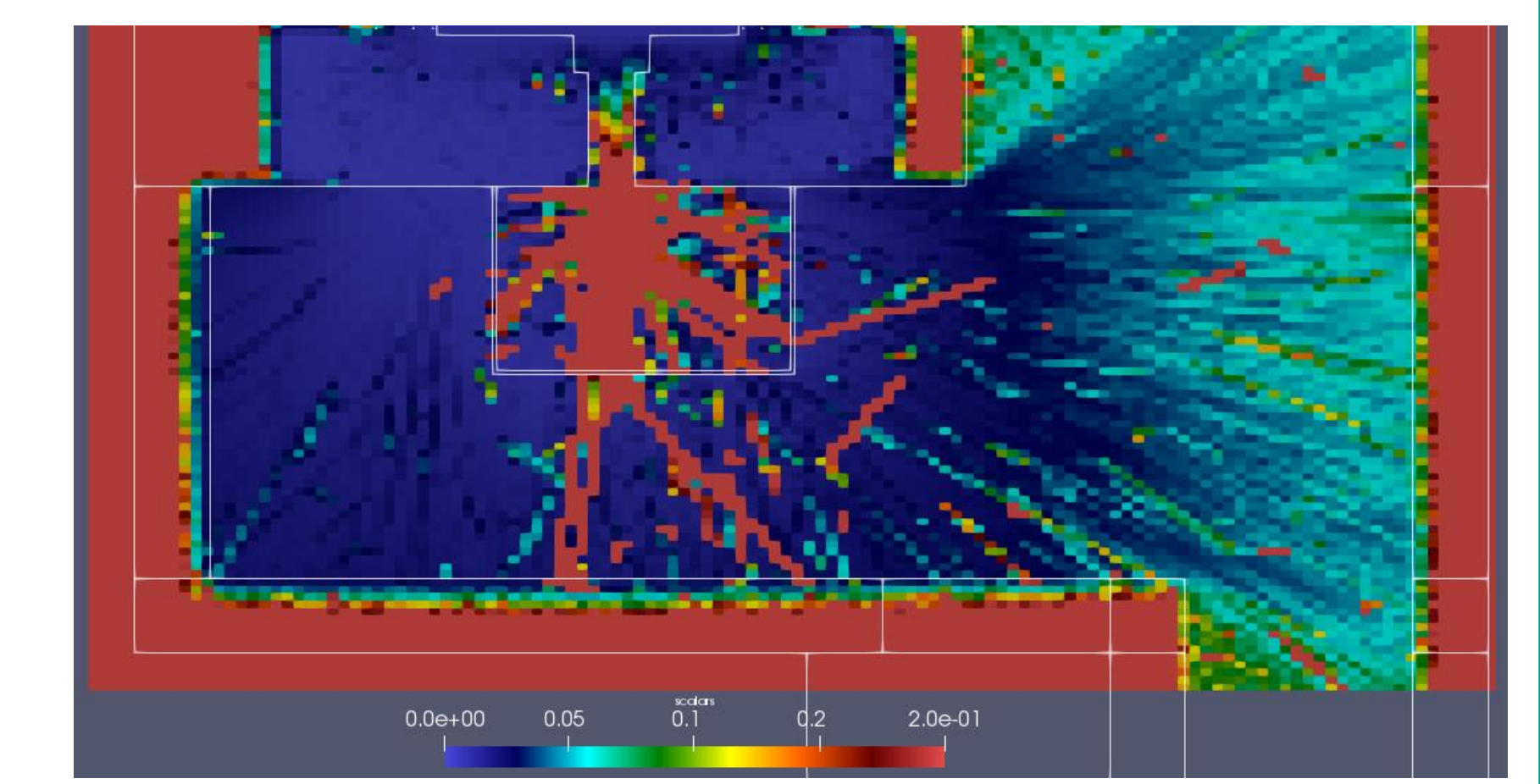

Relative error

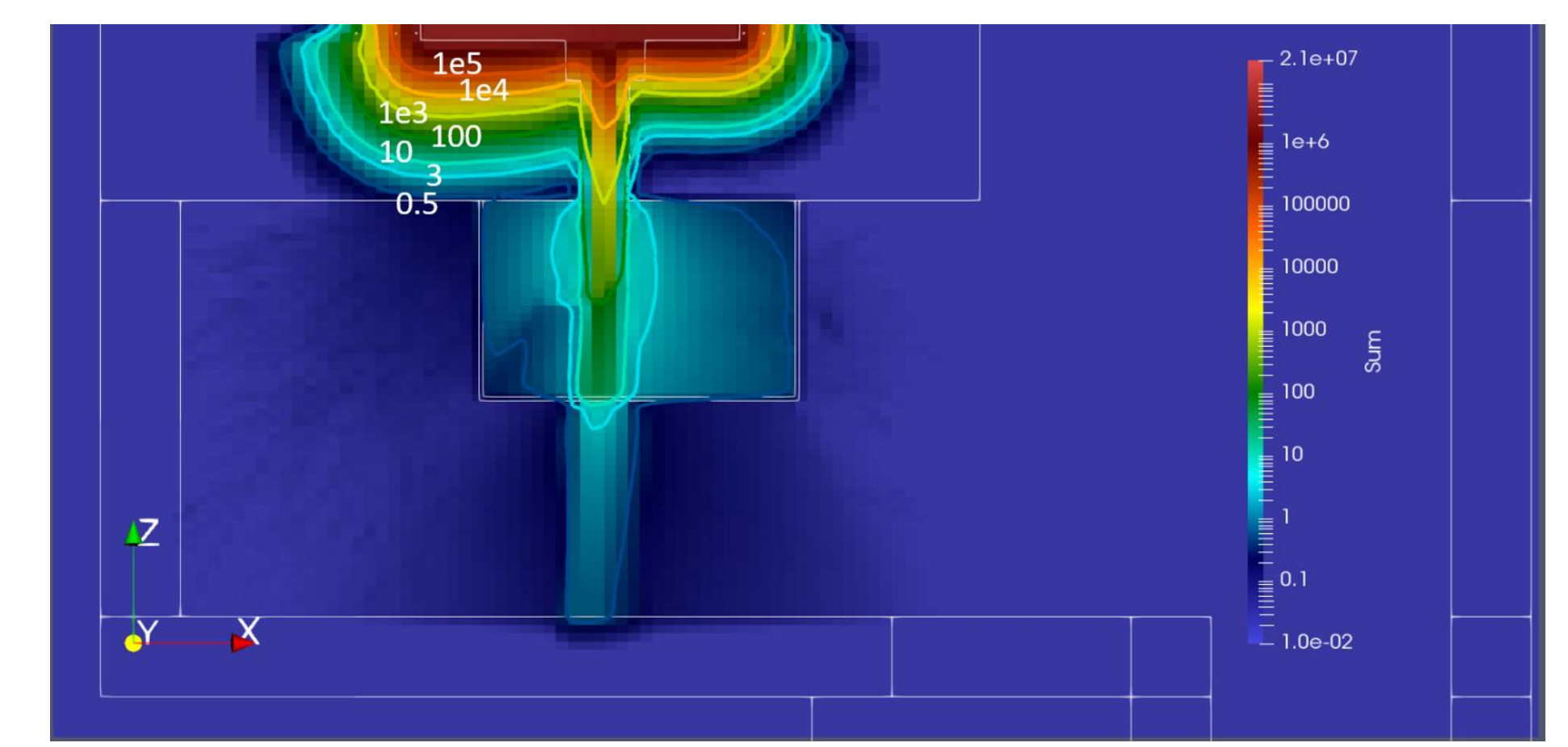

Shutdown dose rate $(\mu \mathrm{Sv} / \mathrm{h})$ with variance reduction

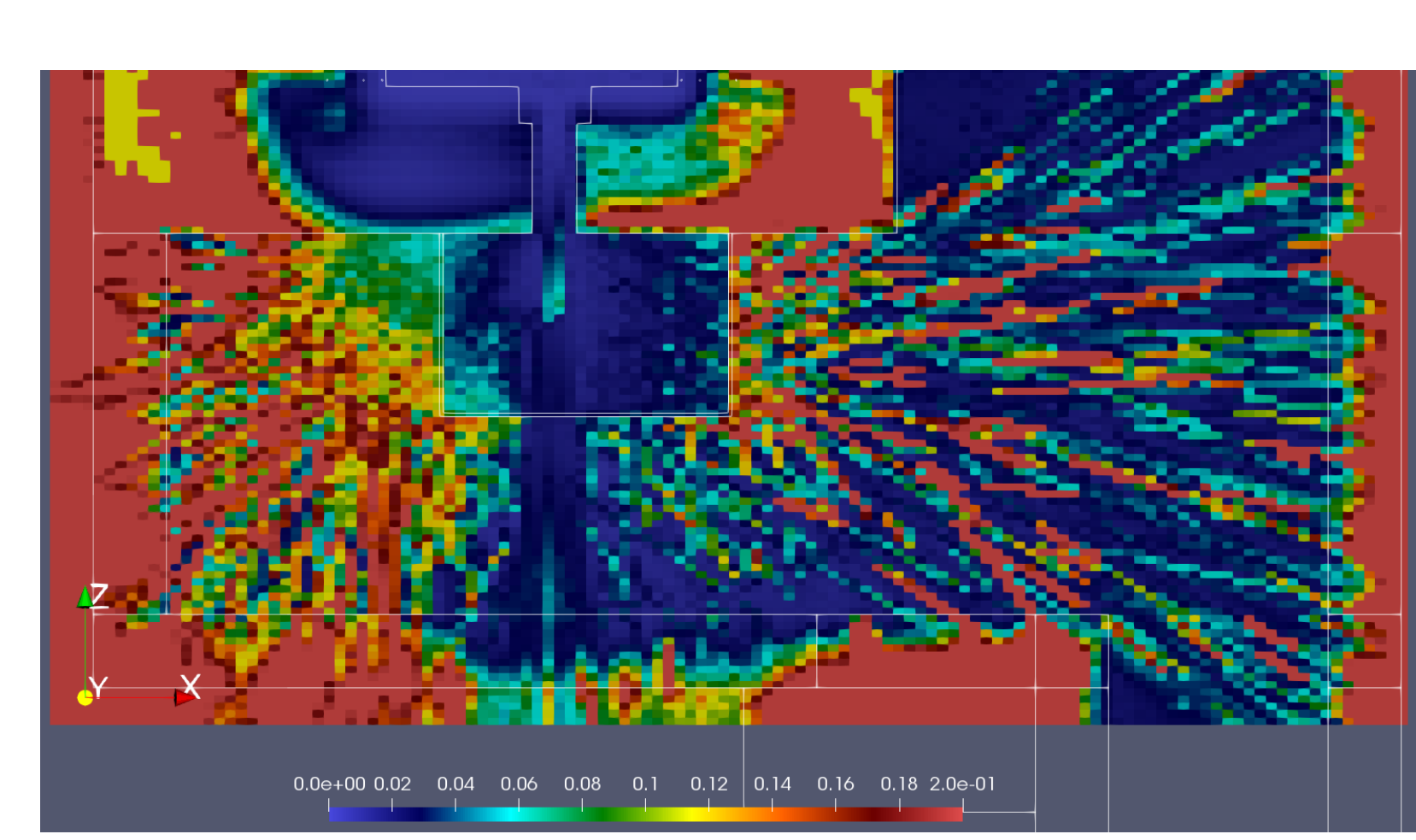

Relative error

\section{Summary}

- The shutdown dose in the LLC has been calculated using new R2Smesh code and a variance reduction approach.

- New version 3.0 of R2Smesh code shows good efficiency and reliability in the verification comparison.
- Weight window mesh generated using ADVANTG code has been used together with an intensity based source sampling.

- The dose in the LLC is $<0.5 \mu \mathrm{Sv} / \mathrm{h}$ for most of the region except TLIC. 